Rahts, Wissenschaftliche Arbeiten des Jahres 1904, betr. Krebsleiden.

Rabaioye, Dégónérescence cancroidale des vieilles actinomycoses. Thèse de Lyon. 1904.

Sanfelice, Die Aetiologio der malignen Gesehwülste. Centralbl. f. Bakteriologie. No. 4.

Schmidt, Herpetische Eruptionen als Vorstadium eines Hautcarcinoms. Arch.

f. Dermatologie u. Syphilis. Bd. 70, Heft 2 (s. a. diese Zeitschr. Bd.II, S. 398).

Snow, Cancer facts and cancer fallacies. Lancet. No. 4229, 17. Septbr. S. 822.

Spude, Die Uxsache des Krebses und der Geschwülste im allgemeinen. Berlin

(Gose u. Tetzlatf) 1904.

Theilhaber u. Hollinger, Die Beschaffenheit des Mesometriums bei Uteruscarcinom. Archiv f. Gynäkologie. Bd. 73, Heft 1.

Ziegler, Salzsäurehyperacidität im Beginn des Magerkrebses. Zeitschr. f. klin. Medizin. Bd. 53.

\title{
II. Arbeiten, betr. die Verbreitung der Krebskrankheit und die Häufigkeit ihres Auftretens. Statistische Untersuchungen.
}

Jieck, Statistik des inoperablen Uteruskrebses. Monatsschrift f. Geburtshülfe u. Gynäkologie. Bd. 20, Heft 2.

Sodré, Die Häufigkeit der Krebskrankheit in Brasilien. Vergl. diese Zeitschr. Bd. 2, S. 407.

Tietze, Statistik der operativen Behandlung des Mammacarcinoms. Therap. Monatshefte. Juli 1904.

\section{Arbeiten, betr. den Krebs einzelner Organe. Kasuistik. Sogen. Krebs bei Tieren und Pflanzen.}

Almeida, Un caso de cancer do estomago. Revista medic. de Sao Paulo, 15. Mai 1904.

Bard, Les formes cliniques du cancer de I'estomac. La Sem. méd. No. 34.

Bidlot, Der K'rebs der Prostata. The scalpel. August 1904.

Bloch, Cancer primitif de la pleure. Bulletin de la société anatom. de Paris. März 1904.

Bodin, Epithéliomes cutanées à évolution très lente. Bull. de la soc. scient. et méd. de l'ouest. XIII. 1. 1904.

Boddaert, Ein Fall von Blasencarcinom. Bull. de la soc. méd. de Gand. Juni 1904.

Coplin, Carcinoma of the oesophagus. Americ. Medicine v. 14. Mai.

Coppez, Un cas de carcinome métastatique des deux chorioides. Arch. ophth.

Februar 1904.

Curtis, Deux cas de cancer secondaire du coeur. Echo méd. du Nord. Juli 1904. Ehrlich, Cylinderzelliges über die Grenzen der Mukosa nicht hinausgehendes

Magencarcinom. Chirurgija. Bd. 15. No. 87.

Galli, Gewichtsunahme bei Magencarcinom. Clinica medica ital. No. 5́.

r. Hansemann, Durchbruch eines Aortenaneurysma in einen Oesophaguskrebs. Sitzung der Berliner med. Gesellsch. v. 13. Juni 1904. 